\title{
Carbon Contamination Topography Analysis of EUV Masks
}

\author{
Yu-Jen Fan', Leonid Yankulin', Petros Thomas', Chimaobi Mbanaso', Alin Antohe', Rashi Garg ${ }^{\prime}$ \\ Yunfei Wang', Thomas Murray ${ }^{1}$, Andrea Wüest ${ }^{2}$, Frank Goodwin ${ }^{2}$, Sungmin Huh $^{2}$, Aaron Cordes ${ }^{2}$ \\ Patrick Naulleau ${ }^{3}$. Kenneth Goldberg ${ }^{3}$, Iacopo Mochi ${ }^{3}$, Eric Gullikson ${ }^{3}$ \\ Gregory Denbeaux \\ 1. College of Nanoscale Science and Engineering, University at Albany SUNY, Albany, NY \\ 2. SEMATECH, Albany, NY \\ 3. CXRO, Lawrence Berkeley National Laboratory, Berkeley CA
}

\begin{abstract}
The impact of carbon contamination on extreme ultraviolet (EUV) masks is significant due to throughput loss and potential effects on imaging performance. Current carbon contamination research primarily focuses on the lifetime of the multilayer surfaces, determined by reflectivity loss and reduced throughput in EUV exposure tools. However, contamination on patterned EUV masks can cause additional effects on absorbing features and the printed images, as well as impacting the efficiency of cleaning process. In this work, several different techniques were used to determine possible contamination topography. Lithographic simulations were also performed and the results compared with the experimental data.
\end{abstract}

Keywords: EUV, mask, carbon contamination

\section{INTRODUCTION}

Carbon contamination on patterned EUV masks is one issue that still must be resolved before EUV lithography is introduced into high volume manufacturing (HVM). It occurs when mask surfaces are exposed to EUV radiation in the presence of residual hydrocarbons. Current solutions are either to reduce the contamination rate and/or to perform a postcleaning process on contaminated surfaces. Our previous work showed that the topography of the contaminated features is critical to estimate the effects of carbon contamination. Possible contamination topographies from a patterned mask are shown in Figure $1^{[1]}$

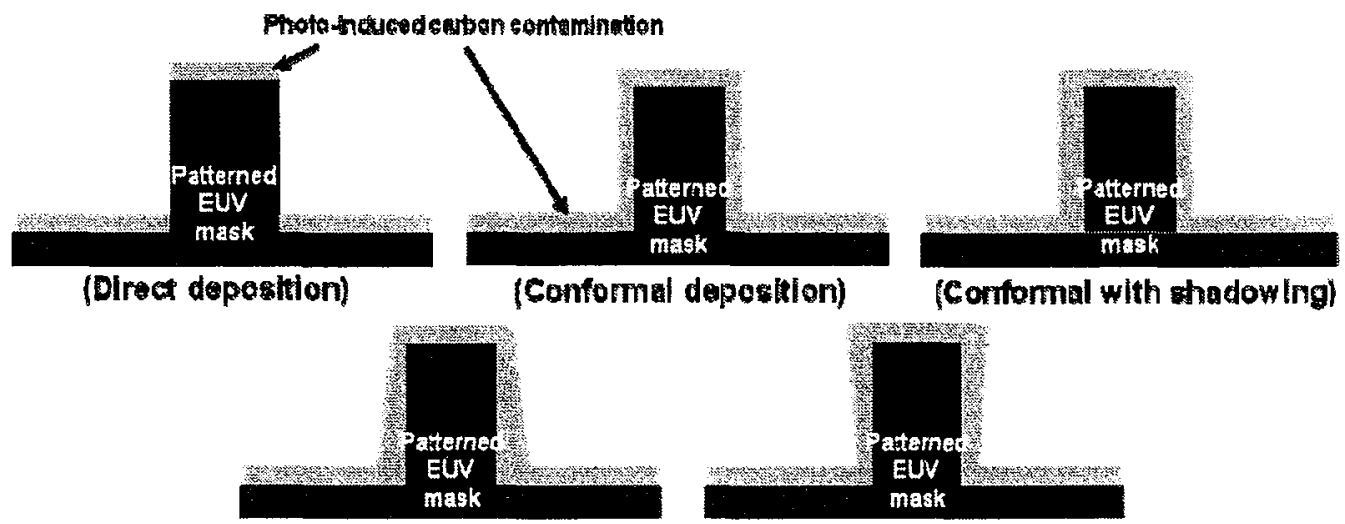

FIG. 1. Different shaped contamination topographies can cause throughput loss, CD change. and dose shift. as well as impacting the efficiency of cleaning process. 
The EUV Microscope for Mask Imaging and Contamination Study (MiMICS) tool was built at the College of Nanscale Science and Engineering (CNSE) to study contamination. ${ }^{[!-3]}$ Selected fields on the mask were contaminated in the EUV MiMICS and analyzed with different tools, including a reticle scanning electron microscope (SEM) for top-down inspection, the SEMATECH Berkeley microfield exposure tool (MET), ${ }^{[4]}$ the SEMATECH Berkeley actinic inspection tool (AIT), ${ }^{[5]}$ and an atomic force microscope (AFM).

Lithographic simulations were performed based on the printing results to understand the contamination topography on EUV masks. Using previously determined resist parameters, aerial images were calculated and compared to the experimental data. The simulated results of contamination topography were used to predict carbon thickness that can be allowed when optical correction is applied to the mask.

\section{SYSTEM OVERVIEW}

A schematic of the EUV MiMICS tool is shown in Figure 2. This tool was designed to study the effects of carbon contamination on the imaging performance of EUV masks. It is equipped with an Energetiq EQ-10M xenon-based discharge-produced plasma EUV source. ${ }^{[6]}$ The incoming beam reflects off a multilayer mirror at $42^{\circ}$ and illuminates the mask at a $6^{\circ}$ off-axis angle of incidence. This geometry allows $13.5 \mathrm{~nm}(92.5 \mathrm{eV})$ EUV in-band radiation and some longer wavelength up through visible light. Photon energies lower than the bond energies of these carbon-containing molecules are not thought to contribute to the contamination. In order to accelerate carbon deposition rate, local pressure was increased to a level allowing experiments to be performed within a reasonable timeframe. Carbon-containing species were then injected into the vacuum chamber using a needle valve. A $3 \times 5 \mathrm{~mm}$ oval aperture was used to contaminate only certain features within a selected area.

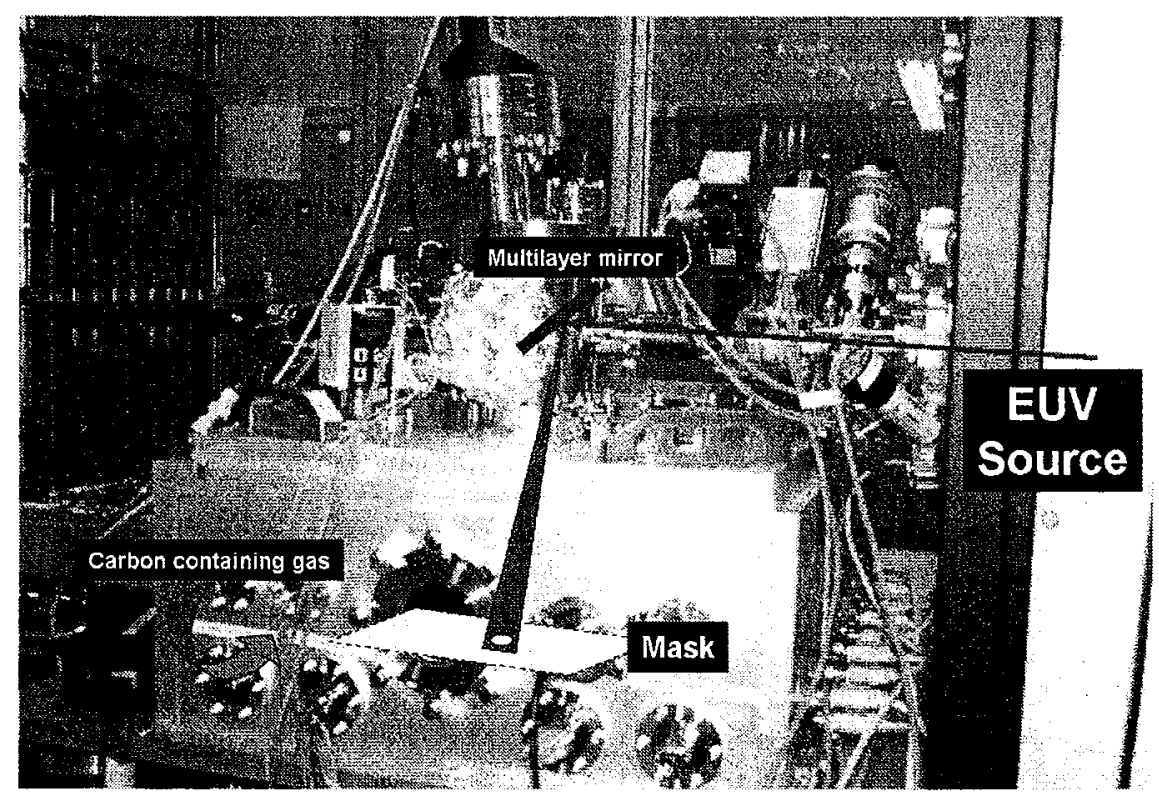

FIG. 2. Layout of the EUV MiMICS at CNSE. Carbon-containing molecules were injected into the vacuum chamber to increase the contamination rate

The film stack used for this experiment was a Si-capped multilayer mirror with $\mathrm{SiO}_{2}$ as a buffer layer and $\mathrm{TaN}$ as an absorber layer. The stack contains 25 fields with different design features including 80 to $225 \mathrm{~nm}$ vertical and horizontal dense lines ( 16 to $45 \mathrm{~nm}$ on the wafer plane when printed in an exposure tool with a demagnification of 5 ). In addition to the dense 1:1 lines, the duty cycles of the lines and spaces vary from $1: 4$ to $4: 1$. Unique labels were also added for easy access to and identification of each field during mask inspection. 


\section{EXPERIMENTAL RESULTS AND ANALYSIS}

Selected fields on the mask were contaminated using the EUV MiMICS tool. The background pressure in the clean chamber was $5 \times 10^{-7}$ Torr and increased to $3 \times 10^{-6}$ Torr with carbon-containing gas injected. The accumulated nominal dose for a typical 8-hour exposure is $5 \mathrm{~J} / \mathrm{cm}^{2}$ including EUV in-band and some longer wavelengths. The carbon density was assumed to be $1.5 \mathrm{~g} / \mathrm{cm}^{3}$ throughout this paper based on current literature reviews from 0.8 to $2.2 \mathrm{~g} / \mathrm{cm}^{3}$. ${ }^{[7-8]}$

\subsection{Mask Inspection}

A top-down reticle scanning electron microscope (SEM) and SuMMIT software were used to record and analyze CD changes on the same field before and after contamination to determine the $\mathrm{CD}$ variation of the absorbing features. ${ }^{[9]}$ The $C D$ change (labeled as $\triangle C D$ ) and the reflectivity loss were measured on the selected fields and recorded in Figure 3 . We observed that carbon contamination results in a reflectivity loss and $\triangle C D$, which implies carbon deposition on the sidewalls of the absorbers.

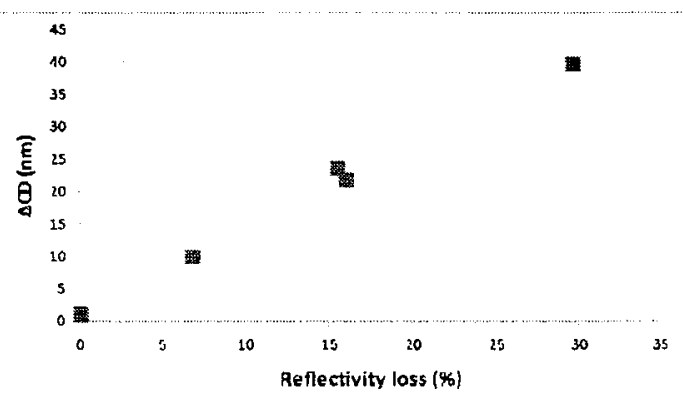

FIG. 3. The CD change was measured as a function of reflectivity loss using a reticle SEM. We observed the 1 CD scales with the reflectivity loss measured from the multilayer surface.

\subsection{Features Printing}

Images were printed at the SEMATECH Berkeley MET; the exposed wafers were analyzed using a CD-SEM. To investigate the impact of carbon contamination on printing, selected fields on the mask were exposed to ELV radiation with the dose controlled to deposit carbon layers of different thicknesses on each field. Carbon thicknesses were determined to be 8,19 , and $40 \mathrm{~nm}$, respectively, based on the CXRO data and reflectivity measurements before printing. ${ }^{[10]}$ For a given field on the mask, Figure 4 compares the relative increase in dose to print $40 \mathrm{~nm}$ half-pitch dense lines as a function of carbon layer thickness with the relative reflectivity loss for the same carbon thickness. For instance, with $19 \mathrm{~nm}$ thick carbon, the dose increased more than $45 \%$ while the reflectivity loss was only $16 \%$. This result indicates that the change in dose depends on not only the carbon thickness, and hence reflectivity loss on the mask surface. but also the topography of the contaminated features.

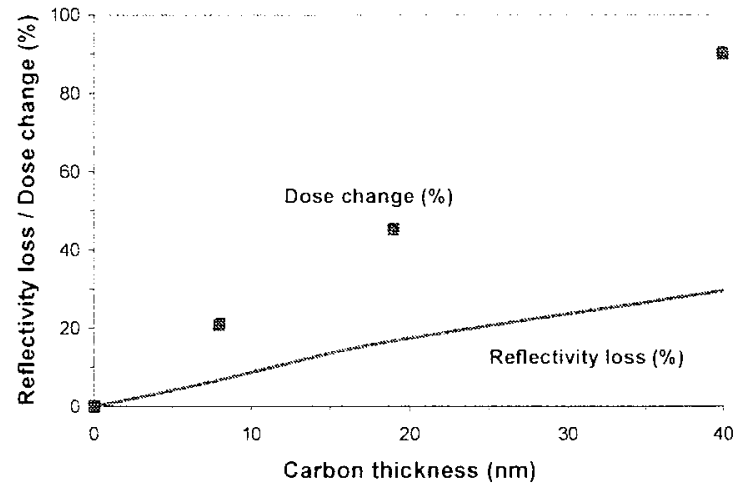

FIG. 4 The required dose change to print dense lines $1 \mathrm{~s}$. carbon thickness is ploted and compared to the measured reflectivity loss on the selected fields of the mask. Carbon thicknesses were determined by transmission efficiency from the CXRO website. 


\subsection{Aerial Image Analysis}

The SEMATECH Berkeley AIT was used to inspect the contaminated mask and record aerial images. The images underwent aerial image data analysis of through-focus behavior and processing such as CD measurement, contrast curve, normalized image log slope, line width roughness, and process window using ThroughFocus software. ${ }^{[1]}$ Figure 5 compares $200 \mathrm{~nm}$ dense lines on both clean and contaminated regions of the mask, which are recorded as aerial image profiles in Figure 6. The thickness of the carbon deposited on the contaminated mask was $19 \mathrm{~nm}$ after ELV exposure in the MiMICS tool with a $5 \mathrm{~J} / \mathrm{cm}^{2}$ nominal dose.
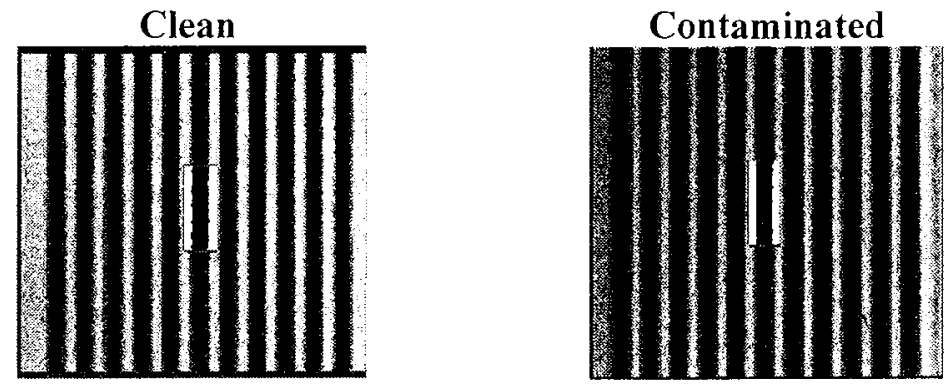

FIG. 5. Aerial images recorded using the SEMATECH Berkeley AIT show $200 \mathrm{~nm}$ dense line features on the mask for the clean and contaminated areas. The $0.5 \mu \mathrm{m} \times 2 \mu \mathrm{m}$ boxes shown were selected for data analysis.

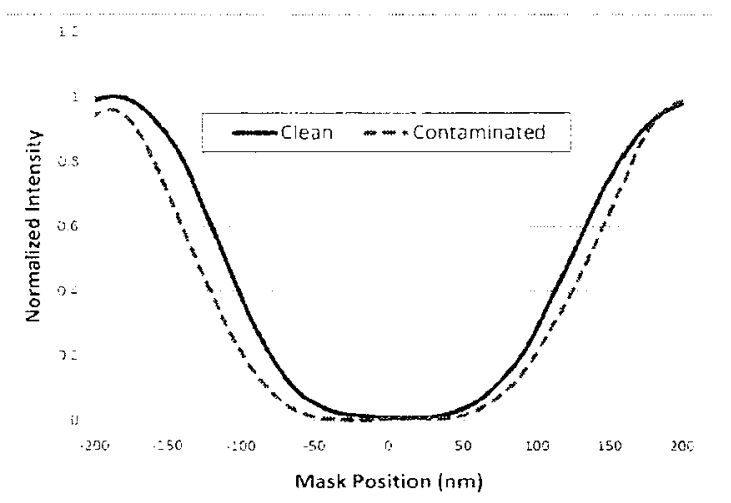

FIG. 6. The intensity profile was recorded based on the location of imaging processing on the mask. Results show an increased CD on a contaminated feature.

\subsection{Surface Analysis}

A Veeco Dimension 3100 AFM with a high aspect ratio focused ion beam (FIB) tip was used in tapping mode to measure surface topography. The full width at half maximum (FWHM) for the contaminated features increased from $234.8 \mathrm{~nm}$ to $275.4 \mathrm{~nm}$, which is a $40.6 \mathrm{~nm} \mathrm{CD}$ change with $19 \mathrm{~nm}$ of carbon deposited on the selected fields of the mask. The root mean square (RMS) roughness of the contaminated region was measured to be $0.45 \mathrm{~nm}$ as compared to $0.29 \mathrm{~nm}$ in a clean area. The increase of RMS roughness can cause more flare during exposure and could be a potential cause of line edge roughness (LER) on the printed images. ${ }^{[12 \cdot 13]}$

A high precision Veeco InSight 3D-AFM was also used to better understand the contamination topography. With the capability of monitoring changes in the tip width, the tip profile can be recorded before and after the measurement, as shown in Figure 8. The cross section profile can then be mapped out and provide an indication of contaminated absorbing features. More carbon was measured near the top corner of the absorbers after contamination. This nonuniform shape could affect the printing performance more than a simple absorption model, as well as causing more throughput loss. However, this cannot yet be considered conclusive until the carbon layer can be measured directly. 

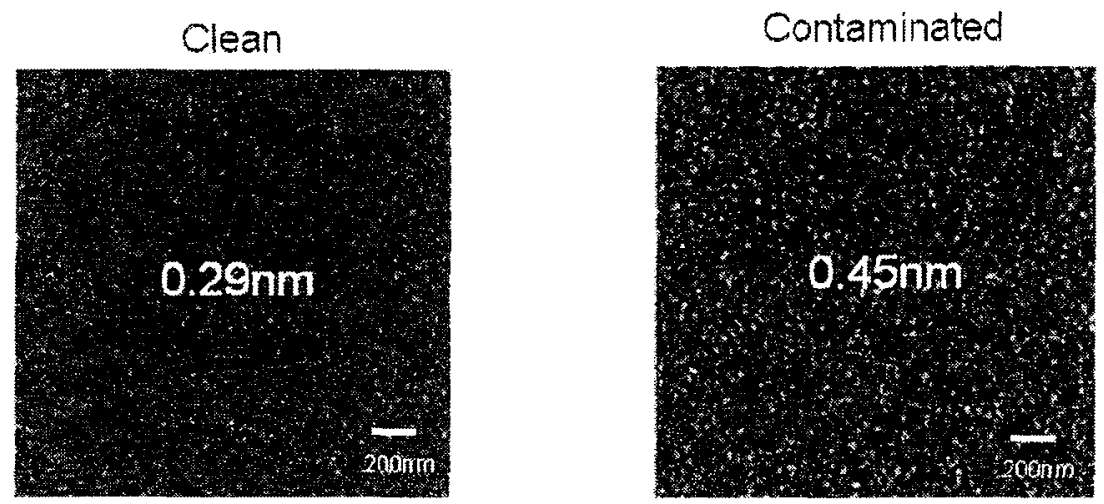

FIG. 7. RMS roughness was increased by approximately $50 \%$ on contaminated region with $24 \mathrm{~nm}$ of carbon deposited.
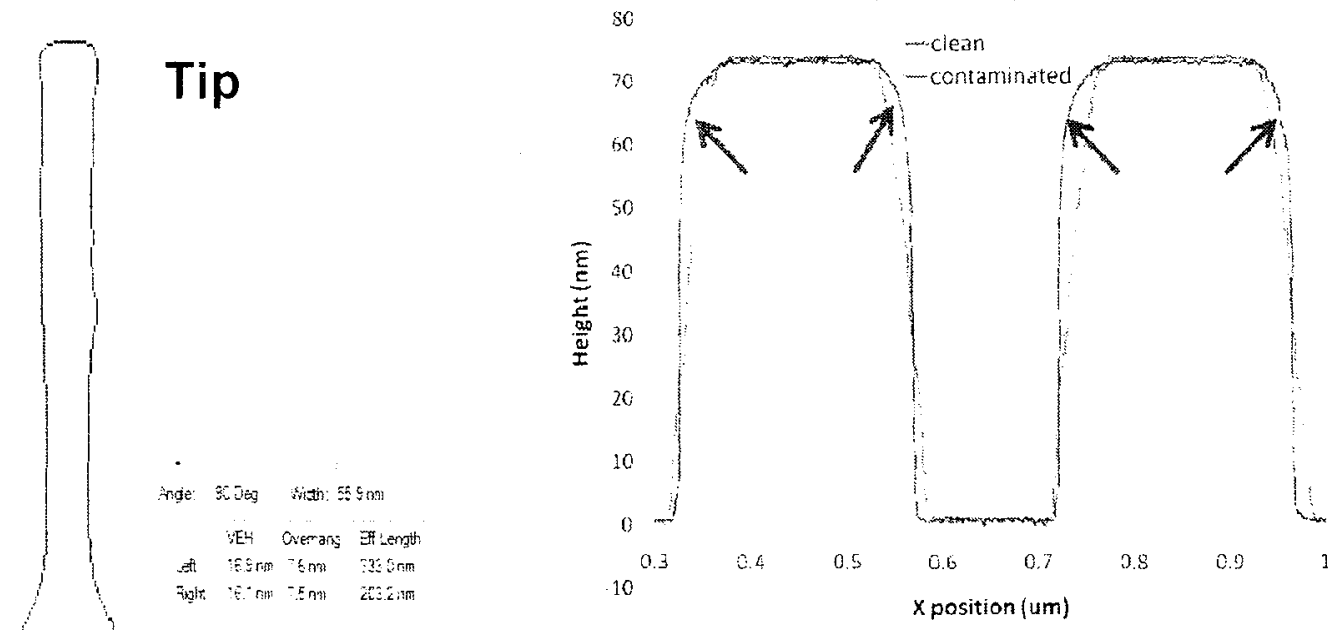

FIG. 8. A tip profile with detailed information indicates the width change before and afier the measurement. With capability to monitor tip wear, the cross-sectional profile of contaminated features can be recorded and compared to the clean features on the mask.

\section{SIMULATION OF CONTAMINATION TOPOGRAPHY}

\subsection{Model Development}

EM-Suite, lithographic software developed by Panoramic Technology Inc. ${ }^{[4.15]}$ was used to investigate the change in the topography of a patterned EUV mask from carbon contamination. It is not yet clear whether the growth rate of the deposited carbon depends on the exposed material such as TaN absorber versus Si or Ru capped multilayers. However, the absorber topography and the illumination angles can still cause different carbon deposition rates between the top surfaces and the sidewalls of the absorbing features. Two extreme cases of possible topography were considered, direct deposition and conformal topography, as shown in Figure 9. Both conformal and direct carbon deposition cause a reflectivity drop due to the absorption of ELV radiation, but the carbon growth on the sidewall in conformal topography increases CD more than direct deposition. 

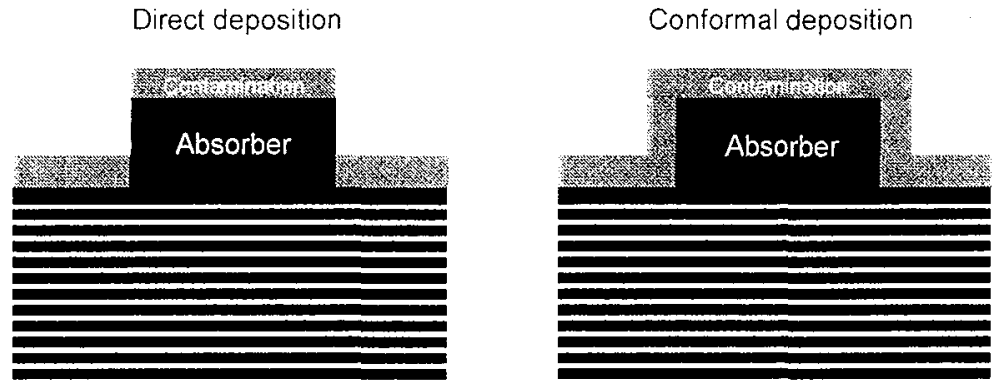

FIG. 9. Two possible topographies: direct and conformal deposition. The film stack used in the simulation consisted of 40 bilayers of $\mathrm{Mo} / \mathrm{Si}, 10 \mathrm{~nm}$ of $\mathrm{SiO}_{2}$ as a buffer laver, and $70 \mathrm{~nm}$ of $\mathrm{TaN}$ as an absorber layer. The gray layer shown in the schematic view represents the deposited carbon layer.

\subsection{Simulation Results}

To understand whether our assumptions matched the experimental data, aerial images and calculated resist parameters were compared to the printed results. As shown in Figure 10, the non-uniform shape was based on our best known topography measured by AFM. Two other possible contamination topography shapes, direct and conformal deposition, were also included. Simulation results show that conformal deposition requires more than $45 \%$ more dose to print the target $C D$ compared to a clean area printing and that non-uniform deposition is even worse which means the contamination topography affects the throughput significantly.

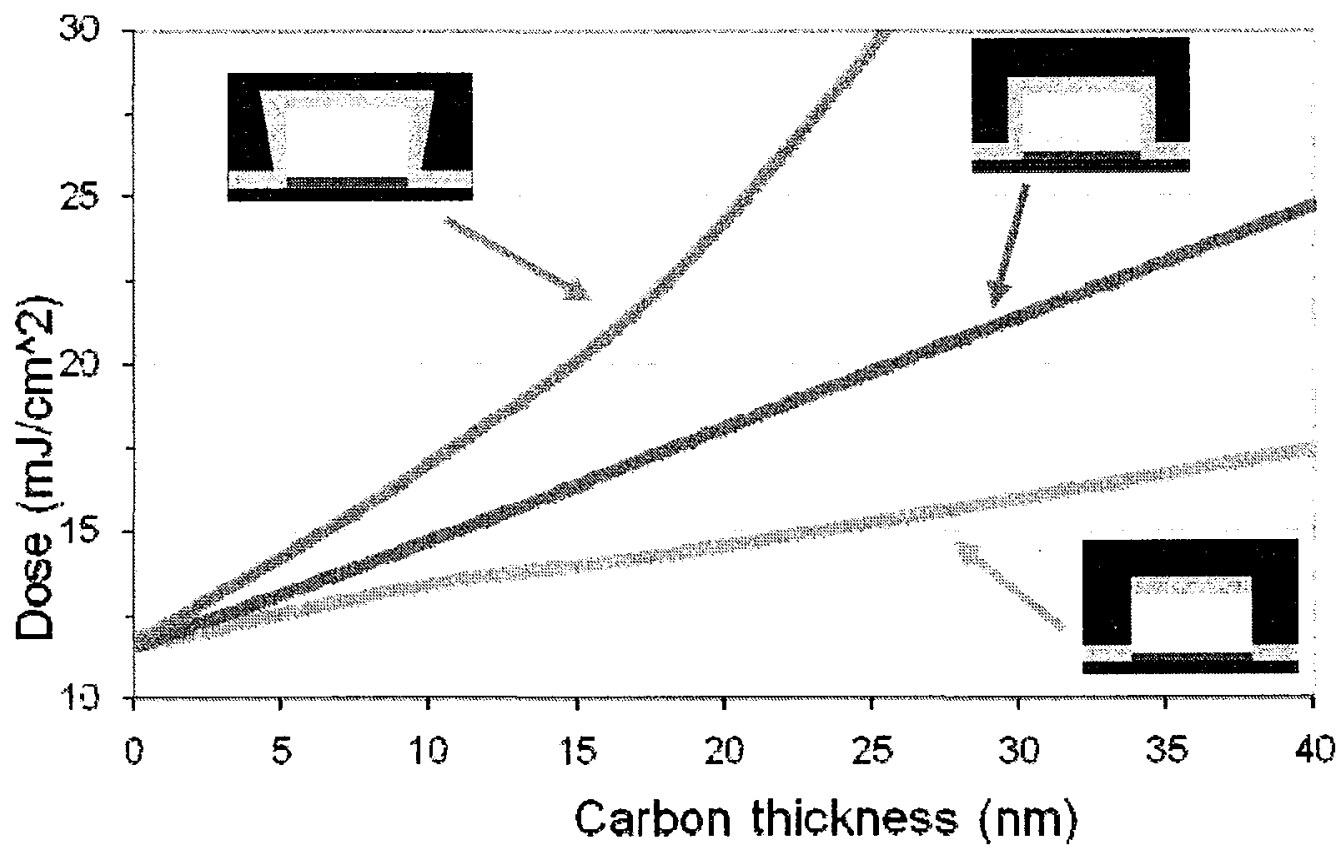

FIG. 10. Simulations were based on our best known topography and compared to direct deposition and conformal deposition.

In actual HVM exposure tools. smaller features. various duty cycles, or unwanted effects such as optical aberrations could make effects of carbon contamination more noticeable even when the carbon layer is thinner. Carbon mitigation

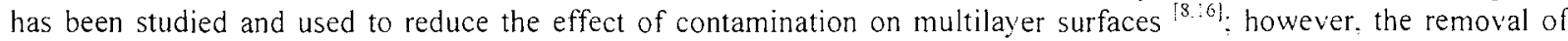
contamination on patterned ELV masks needs more study since the absorbing features can be damaged and affect the lifetime of the mask during the carbon mitigation process. 


\section{CONCLUSION}

The lifetime of EUV optics and masks is critical to move EUV lithography from development into HVM. One of the lifetime issues for EUV masks is EUV-induced carbon contamination.

In this work, selected fields on the mask were contaminated and inspected using a top-down SEM and an AFM. Results showed CD changes in contaminated regions on the mask, which indicate the carbon layer also deposited on the sidewall of the absorbers. Due to the oblique illumination in EUV exposure tools, the sidewall contamination causes extra loss of EUV radiation than a simple absorption model. This was also verified by printing experiments. The required dose was increased dramatically by $45 \%$ when $19 \mathrm{~nm}$ of carbon was deposited. Assuming that the maximum loss in throughput that the industry can tolerate is $5 \%$, which means only $2.5 \mathrm{~nm}$ of carbon contamination is allowable. Although the contamination rate in each exposure tool will be different, it is still likely to fall short of the industry goal of a 30,000 hour lifetime for EUV optics and masks. ${ }^{[7]}$

Aerial image analysis was used to understand the effects of carbon contamination on imaging. Simulations were also performed with both direct and conformal deposition to compare with the best known data. Preliminary simulation results from previous work showed the maximum allowed contamination was $10 \mathrm{~nm}$ before $C D$ compensation failed. ${ }^{i 1}$ However, the predictions of printing performance can be improved only if the actual topography of the carbon contamination is known.

To date, researchers are investigating various techniques to minimize the effects of carbon contamination on EUV optics, such as different capping layers and atomic hydrogen cleaning. ${ }^{[17-18\}}$ For patterned EUV masks, less work has been completed. Direct measurement of the contamination topography is still a challenge, and more work is needed to provide sufficient data to ready EUV lithography for HVM.

\section{REFERENCES}

[1] Y. Fan et al., "Effect of Carbon Contarnination on the Printing Performance of Extreme LItraviolet (ELV) Masks," Journal of liacuum Science and Technology B 28 (2). (2010). To be published.

[2] Y. Fan et al., "Carbon contamination of extreme ultraviolet (ELV) masks and its effect on imaging," Proc. SPIE. Vol. 7271, 72713U (2009).

[3] G. Denbeaux, "ELV mask and optics contamination," International Workshop on ELV Lithography, Hawaii, USA (2009).

[4] P. Naulleau et al., "Status of EUV micro-exposure capabilities at the ALS using the 0.3-NA MET optic," Proc. SPIE 5374, pp. 881-891 (2004).

[5] K. A. Goldberg, P. Naulleau, I. Mochi, E. H. Anderson, S. B. Rekawa, C. D. Kemp, and R. F. Gunion, H.-S. Han, $\mathrm{S}$. Huh, "Actinic extreme ultraviolet mask inspection beyond 0.25 numerical aperture," Journal of lacuum Science and Technology B 26 (6), 2220-4 (2008)

[6] P. Blackborow, M. Partlow, S. Horne, M. Besen, D. Smith, and D. Gustafson, "ELV source development at Energetiq," Proc. SPIE Vol. 6921, 692121 (2008).

[7] J. Hollenshead and L. Klebanoff, "Modeling radiation-induced carbon contamination of extreme ultraviolet optics," J. Vac. Sci. Technol, B 24(1), pp. 64-82 (2006).

[8] Y. Nishiyama, T. Anazawa, H. Oizumi. I. Nishiyama, O. Suga. K. Abe, S. Kagata, and A. Izumi. "Carbon contamination of ELV mask: film characterization. impact on lithographic performance, and cleaning," Proc. SPIE. 6921.692116(2008) 
[9] hup: whit elt. l.com summit

[10] http wwu.cxrolbl.gov:

[11] http: Throughfocus.com

[12] H. Tanabe, G. Yoshizawa, Y. Liu, V. Tolani, K. Kojima, and N. Hayashi, "LER transfer from a mask to wafers," Proc. SPIE. V'ol. 6607, 66071H (2007)

[13] P. Naulleau and S. George, "How will wafer plane line-edge roughness requirements impact mask specifications?" 2009 International Workshop on EUVL, Hawaii, (2009).

[14] http: www panoramictech.com

[15] T. Pistor, "Expanding the Simulation Capability of TEMPEST," MS thesis, Lniversity of California, Berkeley (1997).

[16] S. Huh et al., "Lifetime of EUVL masks as a function of degree of carbon contamination and capping materials," Proc. SPIE. lol. 6921, 692115(2008).

[17] S. Bajt and V. Bakshi, "Optics Contamination," ELV Lithography, SPIE and John Wiley and Sons, Inc.. Chapter 6A. pp. 227-259 (2009).

[18] H Oizumi et al., "Atomic hydrogen cleaning of surface Ru oxide formed by extreme ultraviolet irradiation of Rucapped multilayer mirrors in $\mathrm{H}_{2} \mathrm{O}$ Ambience," Jpn.J. Appl.Phys. Vol. 46. No. 25. pp. L633-L635 (2007).

Supported by the U.S. Department of Energy under Contract No. DE-AC02-05CH11231.

Advanced Materials Research Center. AMRC. International SEMATECH Manufacturing Initiative. and ISMI are servicemarks of SEMATECH. Inc. SEMATECH, and the SEMATECH logo are registered servicemarks of SEMATECH, Inc. All other servicemarks and trademarks are the property of their respective owners. 